\title{
Les ingénieurs et l'éthique professionnelle : pour une approche comparative de la déontologie
}

\author{
Christelle DIDIER
}

Département d'éthique, Université catholique de Lille

Version auteur

REF : Didier C., 2009, «Les ingénieurs et l'éthique professionnelle : pour une approche comparative de la déontologie », in Demazière D. Gadéa C., Sociologie des groupes professionnels. Acquis récent et nouveaux défis, La découverte, Recherche

On rencontre depuis une dizaine d'années en France les termes «éthique de l'ingénieur », « éthique des ingénieurs », " éthique de la profession d'ingénieur » dans la littérature spécialisée, dans des titres de conférences et des intitulés de cours. Ces expressions suscitent en dehors des milieux qui les produisent de la curiosité, de l'étonnement mais aussi parfois du soupçon. L'expression équivalente anglaise engineering ethics ne pose quant à elle pas de problème de compréhension. On la trouve depuis plus de vingt ans dans les dictionnaires de philosophie et d'éthique et sur des couvertures de manuels universitaires qui en sont pour certain à leur quatrième réédition.

L'exercice qui consiste à traduire engineering ethics pour un public français est délicat. Il y a déjà le mot engineering qui est difficilement traduisible de façon satisfaisante. Le terme «ingénierie » que l'on pourrait être tenté d'employer ne désigne pas, en France, l'activité propre des ingénieurs, pas plus qu'il ne délimite les frontières d'une «profession ». Ensuite, le mot ethics est toujours traduit par le mot éthique, en France, alors qu'il devrait parfois l'être par «morale » et dans d'autres cas par «déontologie». Contrairement aux apparences, ce dernier terme ne correspond pas au mot anglais deontology1.

Les auteurs québécois utilisent deux expressions distinctes : dans certains cas, ils parlent d'éthique de l'ingénierie (ou du génie) dans d'autres de déontologie des ingénieurs. Chacune de ces expressions renvoie le lecteur à des champs dont les contours sont clairement

\footnotetext{
1 Le mot français « déontologie » doit être traduit par professionnal ethics, le mot anglais deontology désigne les théories morales fondées sur l'obligation ou le devoir (deon en grec), comme celle de Kant. En français, on parle de déontologisme et de théories déontologiques.
} 
identifiés. Le recours à cet usage aurait pu être une solution pour les chercheurs, enseignants et praticiens français. Cela n'a pas été le cas, peut-être parce que les mots ingénierie et génie ne sont pas employés de la même façon en France qu'au Québec ; sans doute aussi parce que l'utilisation du mot déontologie pourrait être considéré, en France, comme un abus de langage. Il n'existe pas en France, en effet, d'organisation faisant autorité sur les ingénieurs et susceptible de se porter garante de leur déontologie, à l'instar de l'Ordre national des médecins ou celui des architectes.

Cependant, les difficultés de traduction de l'expression engineering ethics ne relèvent pas que d'une question linguistique, d'un problème d'usage des mots. Elles témoignent de différences culturelles et juridiques dont il convient de prendre la mesure si on veut expliquer pourquoi le souci de l'éthique chez les ingénieurs s'est traduit par des réponses dont la forme varie tant d'une région à l'autre du monde. Ce chapitre vise à éclairer quelques aspects de la réalité sociologique qui se cache derrière l'éthique par et pour les ingénieurs. Parcourant tour à tour trois territoires, la France, les Etats-Unis et le Québec, nous étudierons les étapes de la formalisation et de la diffusion dans les milieux professionnels de normes déontologiques produites par et pour des ingénieurs.

\section{Peut-on parler de déontologie au sujet des ingénieurs Français ?}

\subsection{Les deux définitions de la déontologie}

Un «code de déontologie » a été adopté en 1997 par le Conseil national des ingénieurs et scientifiques de France (CNISF). Cependant, certains juristes français considèrent que le recours à ce terme constitue un abus de langage. Selon eux, le terme déontologie renvoie forcément à l'inscription d'un document dans 1'ordre de la loiz et à l'existence d'une instance de régulation identifiée, telle qu'un ordre3. Ces derniers s'appuient alors sur une des visées de la déontologie qui consiste à «édicter des règles suffisamment précises pour être perçues et appliquées comme des impératifs concrets et pour que les comportements fautifs puissent être équitablement identifiés et sanctionnées » (Conseil Supérieur de la Magistrature 2003).

2 Le code de déontologie médicale qui est le texte à portée déontologique le plus connu en France a le statut de décret de loi.

3 L'appellation « ordre professionnel» désigne en France une personne morale chargée d'une mission de service public. Créés par une série d'ordonnances prises en 1945, les premiers ordres «modernes » français, toujours en vigueur en 2008, reçoivent une délégation de la puissance publique pour contrôler la capacité d'exercer certaines activités professionnelles. 
Cette définition de la déontologie rend problématique l'appellation choisie en 1997 par le CNISF pour son code. Elle explique peut-être le changement de nom opéré en 2001 au profit de l'expression retenue depuis de « charte (d')éthique de l'ingénieur »4. A l'époque, les promoteurs de la charte annonçaient que celle-ci se verrait «complétée par la rédaction de plusieurs codes de déontologie de l'ingénieur selon les professions qui [présenteraient], contrairement à [elle] un aspect juridique opposable $» 5$. Le préambule de la charte précisait également cette distinction : "La Charte annule et remplace l'ancien code de déontologie du CNISF. L'appellation « code de déontologie » sera désormais réservée à des documents qui définissent les comportements professionnels corrects dans chacun des métiers d'ingénieurs et dont le non-respect pourrait entraîner l'application de sanctions. » (CNISF 2001). Il ne semble plus être question aujourd'hui de ces «codes de déontologie», probablement parce qu'il manque aux professions ou métiers évoqués dans les documents cités le type d'organisation qui leur permettrait d'imposer le respect d'un code de conduite à leurs membres. Jean Perrin, vice Président du CNISF soulignait déjà en 1997 que le Conseil n'avait pas les moyens de sanctions légaux dont dispose un ordre professionnel, tout en précisant aussi qu'il n'avait pas l'intention de devenir un (Perrin 1997). La question à laquelle le CNISF était confrontée en 1997 reste entière en 2008 : comment imposer un code de déontologie (ou d'éthique) à des personnes qui n'ont pas besoin d'autorisation pour exercer leur métier?

Cependant, il existe une deuxième visée traditionnelle qui voit dans la déontologie non des règles coercitives, mais plutôt «l'affirmation de valeurs indiquant les objectifs à poursuivre et le comportement idéal auquel il faut tendre par un effort constant » (CSM 2003). Le recours à cette acception moins stricte du mot déontologie rend légitime l'appellation « code de déontologie » choisie par le CNISF en 1997. Cette mise en évidence, dans le registre juridique, de deux visée possibles pour la déontologie nous permet de distinguer la dimension normative, voire prescriptive de l'engineering ethics pour laquelle nous réserverons dans ce chapitre le mot déontologie, de sa dimension réflexive pour laquelle nous utiliserons le terme «éthique ». Le mot déontologie renvoie de façon plus évidente au registre des rhétoriques professionnelles - et donc des codes - que ne le fait l'expression « éthique professionnelle ». Cette dernière peut en effet renvoyer à trois types de discours bien différents : normatif, descriptif ou encore réflexif. Dans les pages qui suivent, reprenant à

\footnotetext{
4 Ce document est appelé dans certains document «charte éthique de l'ingénieur » et dans d'autres « charte d'éthique de l'ingénieur », indifféremment.

5 C'est nous qui soulignons. Extrait d'une note de synthèse rédigée suite à la réunion pour la promotion de la Charte organisée par le CNISF le 23-10-2001.
} 
notre compte l'usage québécois, nous utiliserons l'expression « éthique professionnelle » pour évoquer le travail qui consiste à réfléchir aux enjeux éthiques d'une profession ou d'un groupe socioprofessionnel. Ainsi, l'expression «éthique de l'ingénierie » désignera l'étude des questions éthiques que soulève l'activité des ingénieurs.

\subsection{Retour sur l'histoire du code des ingénieurs français}

Ainsi, après dix ans de préparation, un premier code de déontologie pour ingénieurs a donc été publié en France en 1997 (Perrin 1997). Adopté par le conseil d'administration du CNISF, qui représente officiellement les ingénieurs français, ce code changea de nom lors de sa révision. Depuis 2001, la charte d'éthique de l'ingénieur est considérée par le CNISF comme «la profession de foi de tous ceux qui figurent dans le Répertoire français des ingénieurs » (CNISF 2001). Créé en 1998, ce répertoire rassemble des personnes titulaires d'un diplôme d'ingénieur validé par l'Etat français, ainsi que d'autres « reconnues comme exerçant réellement un métier d'ingénieur » par le CNISF. En 2007, le répertoire comportait 380000 inscrits.

Le premier code du CNISF est né de l'adaptation, avec des modifications mineures, du « code de devoirs professionnels » que la Fédération européenne des associations nationales d'ingénieurs (FEANI) avait adopté en 19926. Le comité chargé de proposer un texte pour la FEANI s'était alors inspiré de plusieurs codes extra européens publiés aux USA, au Canada, en Australie et en Nouvelle-Zélande. On peut s'étonner du choix des références exclusivement anglo-américaines quand on sait que plusieurs associations membres avaient déjà publié des codes de déontologie, codes de l'honneur ou encore profession de foi, parfois depuis longtemps. Ce choix s'explique probablement par l'absence de représentants de ces pays dans le comité, où les Français, quant à eux dépourvus de traditions déontologiques, étaient sur-représentés7.

Issu de la fusion d'organisations dont la plus ancienne remonte à 1848, le CNISF est aujourd'hui la seule association représentant officiellement les ingénieurs français auprès des pouvoirs publics. En fait, le CNISF est essentiellement composé de membres indirects par l'intermédiaire de nombreuses associations d'anciens élèves et de quelques sociétés scientifiques et techniques, unions régionales d'ingénieurs et de scientifiques (URIS) et

6 La FEANI est une association européenne d'ingénieurs créée en 1951, par sept pays dont la France. Elle regroupe en 2008 vingt-neuf associations d'ingénieurs à raison d'une par pays et représente près de trois millions et demi d'ingénieurs.

7 Selon Françoise Côme, secrétaire générale de la société européenne des formations d'ingénieurs (SEFI) et déléguée générale de la FMOI (fédération mondiale des organisations d'ingénieurs) (entretien téléphonique datant de janvier 2001) 
sections étrangères. Dans la mesure où ni l'adhésion des associations d'anciens au CNISF, ni celle des anciens élèves à l'association de l'école dont ils sont issus n'est obligatoire, de nombreux ingénieurs diplômés français ne sont pas membres du CNISF. Ceux qui le sont ne semblent pas tellement concernés par ses activités et prises de position officielles. Ils connaissent surtout du CNISF l'enquête sur les rémunérations réalisée régulièrement depuis 1958 et à laquelle 40007 diplômés ont répondu en 2006. Comme dans beaucoup de pays du monde, les ingénieurs français se sentent d'abord parties-prenantes de l'entreprise pour laquelle ils travaillent, avant de se sentir membres d'un groupe professionnel délimité. Si la détention du diplôme reste pour beaucoup d'entre eux - ainsi que dans l'imaginaire collectif national - une marque de distinction, ceux-ci ne se sentent pas pour autant appartenir à un collectif particulier. Les tentations néo-corporatistes, tout comme les discussions autour de la création d'un Ordre des ingénieurs français qui n'ont pas abouti dans les années 1940, n'ont jamais refait surface. Ainsi, la charte (d') éthique de l'ingénieur adoptée en 2001, demeure peu connue de la grande majorité des ingénieurs français.

\subsection{Que dit la Charte (d') éthique des ingénieurs du CNISF?}

Très centrée sur le rôle social de l'ingénieur et sur les enjeux liés au développement durable, la charte comporte quatre parties : «l'ingénieur dans la société », « l'ingénieur et ses compétences », «l'ingénieur et son métier » et «l'ingénieur et ses missions ». Contrairement au code de 1997, il n'est pas fait référence dans la charte à l'usage des titres, au refus des rémunérations irrégulières, à la nécessité de participer aux associations d'ingénieurs8. En revanche, l'ingénieur y est décrit comme s'impliquant dans des actions civiques, transmettant son expérience au service de la société, inscrivant ses actes dans une démarche de développement durable. Bien plus qu'une révision du code, la charte constitue un texte profondément différent, nettement plus ancré que son prédécesseur dans la culture nationale mais aussi dans son époque, avec la référence au développement durable. La charte est plus axiologique que déontologique dans la mesure où elle décrit un idéal vers lequel tendre plutôt qu'elle ne consigne les obligations auxquelles devraient se soumettre les ingénieurs. Elle correspond à la deuxième visée traditionnelle de la déontologie que décrit le Conseil supérieur de la Magistrature, c'est-à-dire à « l'affirmation de valeurs indiquant les objectifs à poursuivre et le comportement idéal auquel il faut tendre par un effort constant » (CSM 2003).

\section{Le cas des Etats-Unis: les codes au cour de la Profession}

\footnotetext{
8 Ce sont des thématiques fréquentes dans les codes of ethics Nord-américains.
} 
Il n'existe pas aux Etats-Unis une association unique rassemblant tous les ingénieurs, et représentant leurs intérêts devant les instances gouvernementales. Il existe en revanche plusieurs associations par branche de métier qui détiennent un statut juridique et occupent une place importante dans l'espace social. Elles constituent, davantage que le CNISF, un lieu de construction des identités professionnelles des ingénieurs. Elles sont toutes dotées depuis longtemps de codes of ethics qui sont régulièrement révisés, renforcés et complétés par des procédures qui diffèrent d'une association à l'autre : analyses de cas publiées dans les revues professionnelles, comité de conseil, ligne téléphonique dédiée, remise de prix mettant à l'honneur des ingénieurs s'étant distingués pour leur professionnalisme. Aujourd'hui, trois codes de déontologie pour ingénieurs sont largement connus et reconnus aux Etats-Unis : celui de l'Accreditation Board of Engineering and Technology (ABET), celui de l'Institute of Electrical and Electronics Engineers (IEEE) et celui de la National Society for Professional Engineers (NSPE).

Aux Etats-Unis comme en France, le port du titre «ingénieur », de même que l'exercice de la profession sont libres. Le respect d'un code de déontologie ne concerne donc que les individus ayant choisi librement d'adhérer à une association professionnelle. Seul les Professional Engineers (PE) font l'objet d'un enregistrement légal9. En 2000, les PEs ne représentaient que $5 \%$ du million et demi d'individus se faisant appeler ingénieurs aux USA 10 (Herkert 2000). Le PE dispose d'un sceau qui est obligatoire pour effectuer certains actes protégés. Il doit être inscrit auprès du Bureau de l'Etat fédéral (State Board) dont il dépend. Ces bureaux qui disposent théoriquement d'un droit de contrôle sur les PE ont tous adoptés des critères déontologiques de conduites professionnelles. Cependant, il convient de nuancer leur pouvoir car ils souffrent d'un important manque de moyens pour mener des investigations auprès des éventuels contrevenants (Harris 1995, 371). Parmi les sanctions disciplinaires enregistrées par le National Council of Examiners for Engineering and Surveying (NCEES) qui fédère tous les State Boards et enregistre les plaintes, on peut lire qu'un PE a vu sa licence suspendue pour quatre ans. Il a du réaliser 400 heures de travail d'intérêt général et suivre un cours de déontologie par correspondance : il avait été convaincu de deux actes de malversation, des détournements d'argent.

\footnotetext{
9 Pour devenir PE, il faut d'abord avoir un diplôme d'ingénieur, passer l'examen des fondamentaux de l'ingénierie (Fundamentals of Engineering), travailler pendant quatre ans sous la supervision d'un PE et enfin réussir l'examen des « principes et pratiques de l'ingénierie » (Principles and Practice of Engineering).

10 C'est-à-dire des détenteurs d'un diplôme universitaire de bac +3 ou 4 , mais aussi des individus moins diplômés mais reconnus comme ingénieurs-maison dans leur entreprise, comme cela peut être encore le cas parfois en France.
} 
La National Society of Professional Engineers (NSPE) a été créée en 1934 pour promouvoir l'idée d'un registre des ingénieurs, aider à la création de State Board dans les Etats où ils n'existaient pas et promouvoir l'adoption de loi créant des licences professionnelles pour les ingénieurs. La NSPE qui avait adopté dans un premier temps le code de déontologie de 1'Engineers' Council for Professional Developpment (ECPD, aujourd'hui ABET), a finalement choisi en 1954 d'avoir le sien et l'a révisé plusieurs fois depuis. La NSPE est dotée d'un comité d'éthique (Board of Ethical Review) qui est le plus actif des Etats-Unis en ce qui concerne les ingénieurs. Les réponses que donne ce comité aux questions éthiques que ses membres lui soumettent, publiées très régulièrement dans la revue Professional Engineer sont même considérées comme une ressource importante par de nombreux chercheurs en engineering ethics (Davis 1998, 46). Les codes des autres associations professionnelles ne font pas toujours l'objet d'une reconnaissance juridique (cela dépend des Etats), mais leur poids symbolique demeure sans commune mesure avec celui de la «Charte éthique de l'ingénieur » du CNISF.

En ce qui concerne l'impact concret des codes de déontologie américains, on peut signaler que le respect de l'un d'eux déjà été cité comme argument dans une cour de justice dans le but de justifier un acte de désobéissance organisationnelle. En 1972, trois ingénieurs travaillant à la réalisation du Bay Area Rapid Transit, un train automatique situé dans la Baie de San Francisco, avaient été licenciés de façon précipitée et abusive. Ils avaient tenté de faire remonter auprès de leur direction leurs inquiétudes au sujet de la sécurité d'un système de freinage conçu par un sous-traitant (Anderson et al 1980). L'IEEE, dont ils étaient tous trois membres, considérant que leurs actions avaient été fondées sur le respect des valeurs de leur profession, et de leur code of ethics, décida de signifier sa solidarité de façon explicite11. L'association déposa au tribunal un Amicus Curiae précisant que tout employeur engageant un ingénieur devrait reconnaître que le respect du code de déontologie de l'association dont cet ingénieur était membre constituait implicitement une obligation à laquelle il était lié dans l'exercice de son activité12. Ces trois ingénieurs furent les premiers récipiendaires du prix Carl Barus décerné par la Société pour l'impact social des technologies de l'IEEE en 1978. Ce prix a été régulièrement décerné depuis.

\section{Le cas Québécois : un ordre professionnel assorti d'un code ayant force de loi}

\footnotetext{
11 Pour plus de détails concernant la politique de IEEE en ce qui concerne l'éthique, cf; Christelle Didier, Penser l'éthique des ingénieurs, PUF, 2008.

12 Extrait de l'interview de John Guarrera, réalisée par Frederik Nebeker pour le compte du Center for the History of Electrical Engineering le 24 février 1995 (source : site web de IEEE, www.ieee.org, consulté le 10/12/2007). John Guarrera était président de IEEE à l'époque des faits.
} 


\subsection{Un contexte particulier d'organisation sociale des professions}

Le contexte réglementaire Québécois est fondamentalement différent des deux précédents. En effet, le système qui gouverne les professions s'inscrit dans une organisation sociale particulière. Cette organisation que l'on retrouve dans les autres provinces et territoires du Canada donne une grande importance aux associations professionnelles dans la construction des liens sociaux et des identités professionnelles. Mais à la différence des EtatsUnis, les corporations ont acquis une reconnaissance très forte des gouvernements des diverses provinces et territoires Canadiens. L'autonomie de la profession d'ingénieur, assortie d'un pouvoir de contrôle de ses membres, est très spécifique au Canada, en tout cas à certaines de ses provinces. Au Québec, cette autonomie est ancienne : la pratique de la profession a été restreinte aux seuls membres de la Société canadienne des ingénieurs civils (SCIC) dès 1898. De 1920 à 1973, la section québécoise de la SCIC porta le nom de Corporation des ingénieurs professionnels de la Province du Québec.

Avec l'adoption par l'Assemblée nationale du Québec du Code des professions, en 1973, les anciennes associations professionnelles provinciales changèrent d'appellation pour s'appeler «Ordres professionnels ». Par délégation de service public, la loi confia aux ordres comme première mission d'assurer la protection du public. Sur le plan administratif, elle donna des pouvoirs plus étendus à leur comités de discipline et fait de leurs codes de déontologie des réglementations ayant quasiment force de loi. Si l'autorégulation est la règle au Québec, il faut préciser que l'autogestion professionnelle a l'obligation de s'exercer dans la forme et la manière prévues dans le Code des professions : 1'autorégulation reste soumise à la surveillance du gouvernement de la Province.

Dans la Province du Québec, il existait en 2007 quarante-cinq ordres professionnels dotés chacun d'une très large autonomie, d'un pouvoir de sanction exercé par un comité de discipline et d'un tribunal professionnel spécifique. La profession d'ingénieur fait partie des vingt-trois professions réglementées à exercice exclusif, c'est-à-dire habilitées à restreindre l'exercice professionnel à leurs seuls membres dans la Province. Deuxième par ses effectifs après celui des infirmiers et infirmières, avec ses 53858 membres, l'Ordre des ingénieurs du Québec a été amené à prendre, en 2006-2007, quarante-trois décisions de type disciplinaire et deux ingénieurs se sont vus révoqués.

En France, seul le titre « ingénieur diplômé de... [nom de l'école habilitée par la Commission des Titres d'ingénieurs (CTI)] » est protégé. Si le contenu des formations est contrôlé par la CTI, la pratique elle-même est libre de même que l'usage du terme 
« ingénieur ». Il en est de même aux Etats-Unis où seul le titre de PE est protégé pas l'appellation engineer. A l'inverse, au Québec, l'usage des mots «ingénieur » et « ingénierie » est réglementé et out abus est sanctionné. En 2005, la Cour d’Appel Québécoise a jugé invalide l'appellation «ingénieur certifié Microsoft »comme traduction de Microsoft certified System engineers (MSCE), que l'entreprise Microsoft avait attribué à près de 35000 personnes ayant suivi une formation interne. L'Ordre des Ingénieurs du Québec porta plainte car il considérait que cette utilisation constituait une usurpation du titre. Suite à la décision de la Cour, Microsoft du payer une amende de 1000 dollars US et surtout renoncer à l'appellation MSCE... en tout cas dans la Province du Québec.

\subsection{Un code de déontologie pour les ingénieurs Québécois}

Chacun des douze provinces et territoires du Canada est doté d'une organisation similaire à celle du Québec pour réglementer l'exercice des professions. Leurs prérogatives, qui peuvent être différentes d'une province à l'autre, se sont toutes vues renforcées d'une façon ou d'une autre au cours des années 1970. Ces associations professionnels et ordres rassemblant les ingénieurs sont regroupés au sein d'une organisation de type fédératif appelé «Ingénieurs Canada (IC)» (ex Conseil canadien des ingénieurs) qui publie, en lien avec l'ensemble de ses membres, des guides généraux assurant une cohérence d'ensemble. Il est doté de son propre code de déontologie, de même que chacune de ses organisations membres. On peut noter que les anglophones utilisent selon les provinces les expressions code of ethics ou encore code of professional ethics tandis que les francophones ont choisi le terme «code de déontologie ». La non-observance du code entraîne des sanctions allant de l'avertissement à la radiation pure et simple, et donc l'impossibilité de pratiquer dans la Province. Certains de ces codes font partie intégrante de la loi provinciale, comme par exemple celui du Québec et de l'Ontario.

Le code de déontologie de l'OIQ, qui a le statut d'une loi professionnelle, est un texte très différent à la fois le la «Charte (d') éthique de l'ingénieur » du CNISF et des différents code of ethics existant aux Etats-Unis. Il est composé de cinq sections constituées ellesmêmes de plusieurs articles, dont une trentaine pour la section III intitulée « devoirs et obligations envers le client $»$. Du fait de la portée disciplinaire et juridique de ce code, la formulation des articles qui le composent est très précise. Pour illustrer ce propos, nous pouvons citer l'extrait des conclusions d'un comité de discipline de l'OIQ datant du 16 mai 2002. Les chefs d'accusations étaient les suivants : «dans le cadre d'un mandat relatif à la conception de l'installation sceptique d'un immeuble (...) l'ingénieur P.C. a exprimé un avis 
qui n'était pas basé sur des connaissances suffisantes et d'honnêtes convictions, contrevenant ainsi à l'article 2.04 du code de déontologie des ingénieurs (...) [il] présenté un plan incorrect contradictoire et/ou insuffisamment explicite, contrevenant ainsi à l'article 3.02.01 » du même code. Le comité de discipline a condamné l'ingénieur à payer deux amendes de 600 dollars canadiens ainsi qu'à payer les «frais et débours de la cause ». Cet usage juridique du code explique l'importance accordée à la formation déontologique des ingénieurs. Cette formation est d'ailleurs requise pour tout ingénieur étranger voulant exercer au Québec. L'obtention du permis d'exercer dépend, entre autres, de la réussite d'un examen professionnel portant sur l'exercice de la profession, la déontologie, ainsi que le droit et les obligations en matière de génie.

\section{Conclusion}

Bien que les trois pays choisis soient confrontés à des enjeux économiques, techniques et sociaux assez comparables, dans chacun d'eux la prise en compte des questions déontologiques dans les milieux d'ingénieurs s'est faite de façons très diverses, dans le fond comme dans la forme. A l'ère de la mondialisation des échanges de savoirs et de biens, la quête de repères éthiques s'effectue plus que jamais dans un contexte de pluralisme des cultures et des valeurs. On peut se demander si les ingénieurs du XXIème siècle parviendront à donner à leurs successeurs des repères qui leur permettront de respecter mais surtout de dépasser les clivages culturels, des repères à la mesure des enjeux de la mondialisation.

Trois pistes de travail émergent du travail présenté dans ce chapitre. Rappelons d'abord la nécessité de clarifier les mots de l'éthique et plus particulièrement ceux de l'éthique professionnelle. De nombreuses incompréhensions de part et d'autre de l'Atlantique reposent sur un manque d'explicitation de termes que beaucoup pensent univoque13. Ce travail nécessaire est en cours pour ce qui concerne certains usages des langues anglaise et française. Mais l'engineering ethics se développe dans d'autres langues et des univers culturels bien éloignés de ceux des Etats-Unis, du Québec ou encore de la France. Des travaux commence a paraître en japonais14, l'association des ingénieurs mécaniciens chinois a promulgués récemment un code d'éthique15.

\footnotetext{
13 Extrait plus qu'éloquent du commentaire qu'un referee a rédigé au sujet d'un autre article sur l'engineering ethics que j'ai proposé récemment pour un ouvrage collectif : « At first reading, I was convinced that the author could not possibly be serious. (...) But as I read the rest of the paper, it became clear that the author was not ill informed, and he was not joking, but had something interesting to say to the rest of us who have always assumed that what we were talking about made perfect sense to others".

14 Citer Jun Fudano et Lueghenbiel

15 Citer source
} 
La seconde leçon tirée de ce travail concerne le statut des documents constituant une formalisation du souci de l'éthique qui traverse la "profession » (au sens large) d'ingénieurs. Il est toujours possible de faire des analyses de contenu, de repérer les thématiques communes à ces textes et d'essayer d'expliquer les variations qui apparaissent d'un lieu à l'autre, d'une époque à l'autre. Mais, il faut garder à l'esprit que chacun de ces documents n'a de sens que dans contexte particulier16. Les codes d'éthique et ou de déontologie écrit par et pour des ingénieurs sont des objets qui ne peuvent pas être abstraits de leur contexte, celui des instances qui les ont produit et/ou qui ont charge de leur faire appliquer, celui aussi de la gestion des relations entre les ingénieurs et leurs employeurs, celui de leur statut juridique....

La troisième leçon concerne la nécessité de prendre en compte les variations d'un territoire à l'autre (pays, région) de la définition du domaine d'action désigné par les termes engineering/ingénierie/génie. Ce travail est nécessaire et important tant les métiers exercés par ceux et celles qui l'ont appellent ingénieurs/engineers varient de part le monde. Cependant, il convient de garder à l'esprit que seule une mise entre parenthèse de cette approche démacrationiste des professions permet d'entrer (enfin) dans le cour des questions qui sont communes à tous les territoires : les problèmes éthiques qui se posent à l'interface des techniques (de leur conception à leur disparition, en passant par leur fabrication et leur diffusion), et du reste du monde animé et inanimé. A trop expliciter les différences d'usages des mots et de position des frontières, on risque de perdre de vue ce qui est largement commun entre tous ces documents : leur

\section{Références bibliographiques}

ANDERSON Robert M., PERRUCI Robert, SCHENDEL Dan E., 1980, Divided Loyalties. Whistleblowing at BART, Purdue University Studies in Science, Technology and Human Values, West Lafayette, IN.

CSM (Conseil supérieur de la magistrature), 2003, Rapport annuel 2003-2004. Consultable a l'adresse suivante : http://www.conseil-superieur-magistrature.fr (consulté le 10 avril 2008)

CNISF (Conseil national des ingénieurs et scientifiques de France), 2001, «Charte éthique des ingénieurs du CNISF ».

16 L'idée de code d'éthique pour ingénieur à portée internationale défendu pendant un temps par Vivian Weil me semble un objectif irréaliste. Récemment la FEANI a renoncé à l'idée d'un code européen pour en revenir à l'idée du code « de la feani ». 
CNISF, 2007, Observatoire des ingénieurs Français. Rapport de la 18ème enquête du CNISF, CNISF, Paris. (consultable en ligne à http://enquete.cnisf.org/2007/enquete_2007.pdf, consulté le 10 avril 2008)

DAVIS Michael, 1998, Thinking Like an Engineer, Study in the Ethics of a Profession, Oxford University Press, Oxford.

DIDIER Christelle, 2008, Penser l'éthique des ingénieurs, Presses Universitaires de France, collection « questions d'éthique », Paris.

HARRIS Charles E. jr, PRITCHARD Michael S., RABINS Michael J., 1995, Engineering Ethics Concept and Cases, Wadsworth Publishing Company, Belmont, réed. 2000.

MITCHAM Carl, 1992, Engineering ethics throughout the world. Introduction, documentation, commentary and bibliography, draft version 1.0, STS Press, Pennsylviana State University.

PERRIN Jean, 1997, «Le code de déontologie de l'ingénieur », in Raymond Moch, dir. Ethique et société : les déontologies professionnelles à l'épreuve des techniques, Armand Colin, Paris, pp. 181-193. 